searching among millions of genomic variants to find those that make a difference in disease susceptibility or in other traits." Among the recipients was Manolis Kellis, Professor of Computer Science at the Massachusetts Institute of Technology (MIT) and a member of the Broad Institute of MIT and Harvard, who is exploiting computational strategies and epigenomics data sets to identify phenotype-causing single-nucleotide variants (SNVs) in non-coding DNA.

During a recent seminar at the American Society for Human Genetics meeting, Kellis laid out six steps required to accomplish that task: identifying $(i)$ the cell and tissue types in which the variant is active, (ii) the target gene(s) through which it acts, (iii) the causal mutation among multiple associated variants, (iv) the DNA-binding protein that mediates the phenotype, and characterizing $(v)$ the cellular and (vi) organismal consequences that result from misregulation of these interactions. Kellis and his colleagues also documented the strategy in a beautiful proof-of-principle study that dissected the mechanism underlying the strongest genetic association with obesity (2). More than 7 years ago, he says, genomewide association studies (GWAS) identified 89 common variants spread across some 47,000 bases in the FTO gene region. However, which variants actually lead to obesity risk, and which are merely along for the ride, has remained unknown-as have the cell type, regulator, target genes, and cellular pathways through which the causal variants act.

To work that out, Kellis and his colleagues tapped into data from the $\mathrm{NIH}$ Roadmap Epigenome Project, of which he was a principal investigator. Comparing chromatin modification states across 127 different cell types, they identified a 12.8-kb "super-enhancer" in the FTO intron that was specifically active in adipocyte progenitor cells. That enhancer is located in a 2-megabase chromosomal compartment, or "topologically associated domain," along with 8 other genes, any of which could be mediating the association. By comparing the activity of those genes in cells with both the risk and non-risk versions of the enhancer, the team identified two target genes, $I R X 3$ and $I R X 5$, which they then showed are responsible for the association.

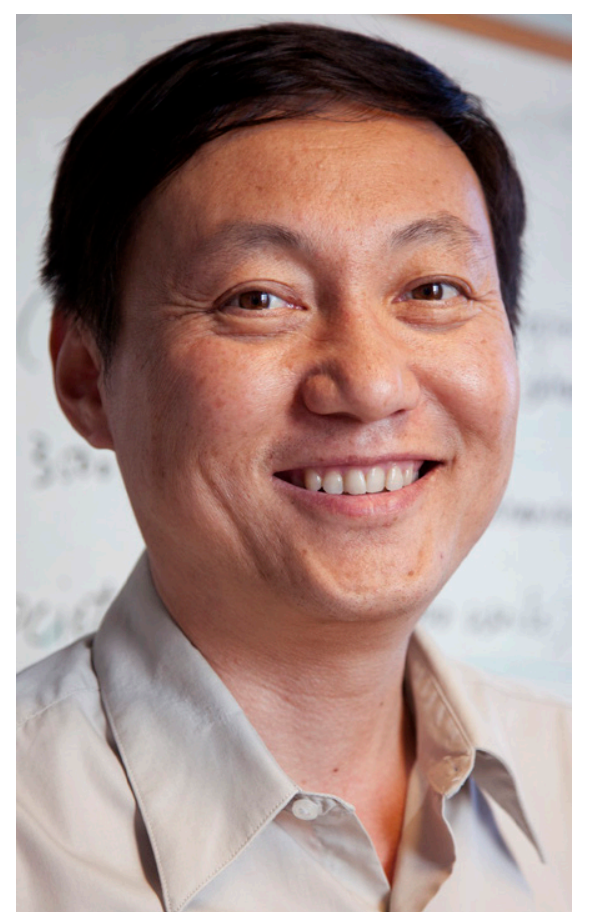

Bing Ren, a Member of the Ludwig Institute for Cancer Research in San Diego, is developing novel computational strategies to identify disease-causing variants located in non-coding regions of DNA. Credit: Ludwig Institute for Cancer Research.

The question then became which nucleotide in that enhancer actually causes the high-risk phenotype. Using a computational approach that takes into account the evolutionary conservation of protein-binding motifs, the team zeroed in on 1 of the $89 \mathrm{com}$ mon FTO variants, a T-to-C change known as rs1421085 that knocks out binding for the ARID5B transcription factor. Using a combination of genetic manipulations including gene overexpression, knock-down, and CRISPR/ Cas9-mediated editing, the team demonstrated that ARID5B binding to the FTO intron represses expression of $I R X 3$ and IRX5, which promote fat storage, keeping the cell in a fat-burning, heat-generating (thermogenic) mode. The high-risk DNA variant derepresses that enhancer, switching the cell from mitochondrially active to sluggish.
Kellis and his colleagues now plan to take their approach high-throughput in the hope of identifying causal variants for hundreds of phenotypes. But he acknowledges it won't be easy since the cellular signatures of organismal phenotypes are not necessarily obvious and may differ greatly between diseases and even for genetic variants associated with the same disease. Even when these cellular signatures are known, measuring them at high-throughput for hundreds of perturbations remains an unsolved engineering challenge. In the case of obesity, they plan to measure cellular respiration rate as an indicator of thermogenesis for dozens of candidate genes and regulators. "Bridging the gap between genetic alterations and organismal phenotypes is one of the greatest challenges of our lifetime," he asserts. "But solving this problem can change our understanding of complex disorders and lead to new treatments, so any investment towards it is well worth the effort."

Bing Ren, a Member of the Ludwig Institute for Cancer Research in San Diego, is also interested in causal variants in non-coding DNA, but he won't be using GWAS to find them. GWAS, Ren explains, have three key limitations: They make no assumptions about disease mechanism, require large cohorts of patients and controls, and yet "capture only a fraction of the heritability that can be recovered."

Ren, along with University of California, San Diego geneticist Kang Zhang and bioinformatician Sheng Zhong, is developing what he calls a "fundamentally different approach," starting with the assumption that many diseases can be traced to alterations in transcription factor binding. By mining epigenomic data sets-specifically, transcription factor binding and histone modification signatures measured using ChIPSeq-the team hopes to identify DNA sequence variants that can explain the different patterns, which they can then use to screen new individuals for disease risk based on their DNA sequences alone.

Ren's team will initially test the approach using a disease with known 
genetic influences, age-related macular degeneration (AMD), before applying it more broadly. "This approach directly addresses the fundamental models of how a disease arises," he explains. "And if we are successful, we would not only identify diseasecausing sequence variations but also learn about fundamental mechanisms of a disease such as AMD."

\section{Folding variations}

Alain Laederach, Assistant Professor of Biology at the University of North Carolina (UNC) at Chapel Hill, is another NHGRI grant recipient. He was awarded \$2.2 million to study the impact of single nucleotide changes on RNA structure, a class of variants that he dubs "riboSNitches."

"It's basically a play on words," Laederach explains - a cross between a riboswitch (an RNA molecule that changes conformation by binding a small molecule) and an SNV. A riboSNitch, he says, is a sequence variant "that leads to either a change in transcription or translation," and they could represent as many as $15 \%$ of SNVs overall.
In 2010, Laederach and his colleagues used a computational algorithm called SNPfold to screen 514 SNVs in the Human Genetic Mutations Database for variants that might impact mRNA secondary structure, ranking them in order of structural disruption (3). They identified seven diseases that could be attributed to such variants, including hyperferritinemia cataract syndrome, a condition caused by excessive translation of ferritin protein in the eye; cartilage hair hypoplasia, caused by riboSNitches in a long non-coding RNA called RMRP that alter a cell's ability to process that transcript into regulatory microRNAs; and retinoblastoma, a cancer of the eye in which folding changes in the $5^{\prime}$ untranslated region of the retinoblastoma protein mRNA impair translation.

"This concept that RNAs fold ... and that mutations can cause them to misfold and, in certain cases, lead to a [change in] phenotype, is the idea of a riboSNitch," Laederach says.

Now Laederach plans to use his NHGRI funding to take his search genome-wide, targeting more common conditions, such as Alzheimer's disease, Parkinson's disease, and cancer. Laederach's team has adopted selective 2'-hydroxylacylation by primer extension (SHAPE), a method developed by UNC colleague Kevin Weeks that employs different chemical reagents to modify either single- or double-stranded RNA, which is then read out using next-generation DNA sequencing. A related method called PARS (parallel analysis of RNA structure), developed in the lab of Howard Chang at the Stanford University School of Medicine, obtains the same information using single- and doublestrand-specific ribonucleases. In one recent study, Chang and his team used PARS to obtain "the initial landscape and variation of RNA secondary structures" from a family trio (two normal parents and their child). Their analysis identified 1907 riboSNitches among the 3 individuals, including 22 "associated with diverse human diseases and phenotypes" (4).

Most transcripts, Laederach explains, are relatively robust to structural variation. "If you change the RNA

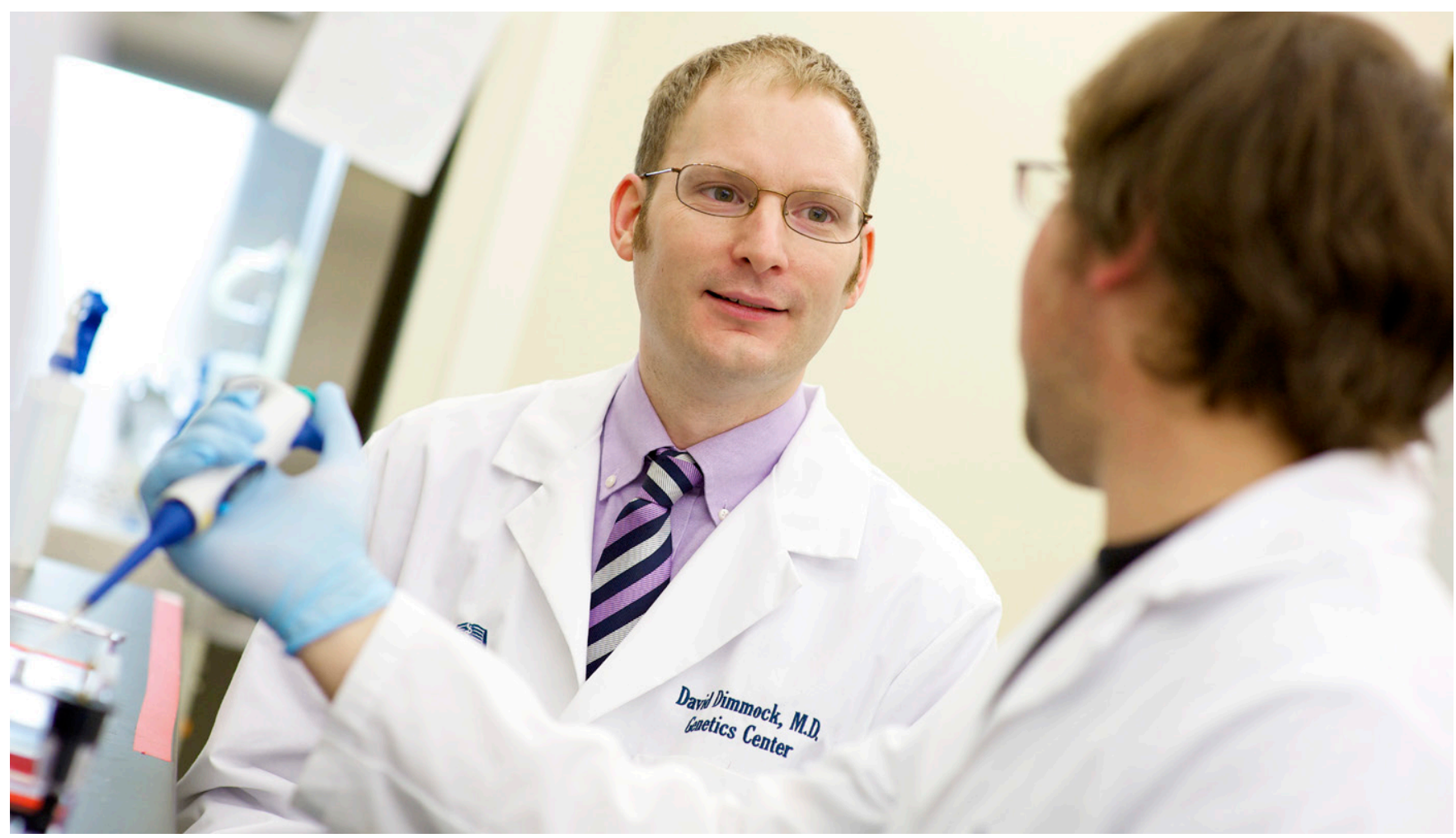

David Dimmock, Associate Professor at the Medical College of Wisconsin, uses clinical and genomic data to inform his diagnoses. Credit: Medical College of Wisconsin. 


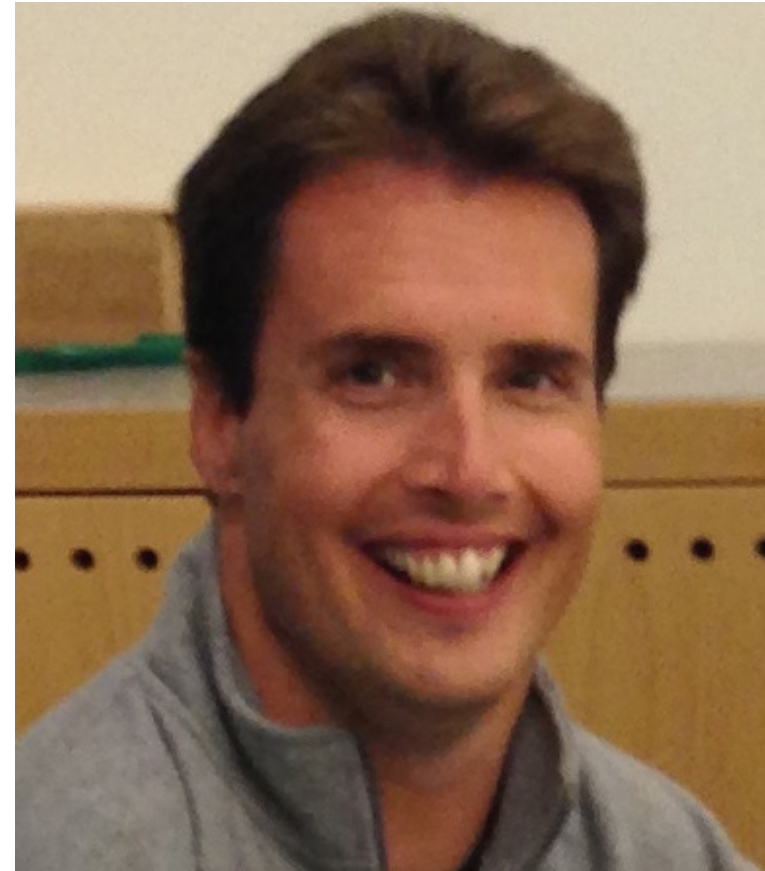

Alain Laederach, Assistant Professor at the University of North Carolina at Chapel Hill, received a \$2.2 million grant to study "riboSNitches." Credit: Silvia Ramos.

\section{Gene Codes Corporation \\ T C A G ENE \\ A G T C O D E S}

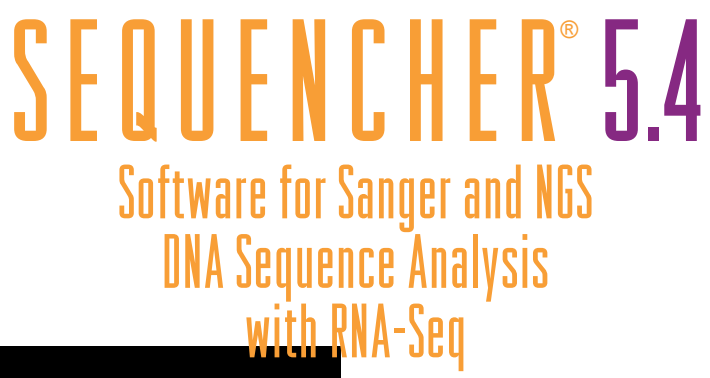

Download today!

visit WWW.genecodes.com

\section{Power with Simplicity}

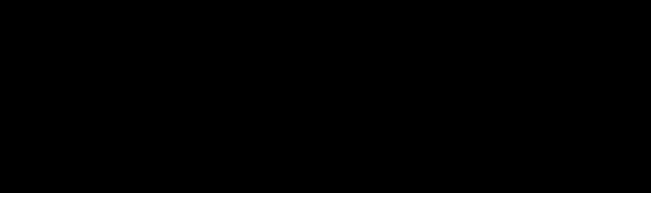

structure, you're not going to die." But, "there are critical regions of our transcriptome that, if the structure is messed with in some way, will lead to a phenotype," and they may reflect the subtle differences in risk that often are associated with such common conditions.

Such data could have therapeutic value, Laederach says. Researchers at Merck recently described a small molecule called ribocil capable of targeting an E. coli flavin mononucleotide-responsive riboswitch and dampening bacterial infection in mice. If one could target ribosNitches in the same way, he says, it might be possible to mitigate the effects of harmful variants in humans. "You could switch the SNitch," Laederach quips.

\section{Using SNV knowledge in the clinic}

At the moment, therapeutics remain far in the future. But there's no denying whole-genome sequencing is increasingly being applied in the clinic, especially for diagnosis of rare pediatric and neonatal abnormalities.

David Dimmock, Associate Professor of Pediatrics at the Medical College of Wisconsin, notes that such studies are distinct from traditional bench research. In the research lab, he says, scientists are free to go wherever the science takes them. In one recent study, for instance, George Church at Harvard Medical School, and colleagues used patient-derived iPS cells, CRISPR/Cas9mediated gene editing, and cultured "organoids" to establish the link between specific mutations in the Tafazzin gene TAZ and a form of congenital cardiomyopathy called Barth syndrome. That study employed a cardiomyocyte contraction assay that measured the ability of cellular sheets to repeatedly curl and unfold like paper (5).

But in the clinic, it isn't always clear what physicians can do with such information. Here, speed is paramount, as even short delays can have deleterious consequences. As a result, clinical geneticists have steadily accelerated their whole-genome sequencing and interpretation services. The Medical College of Wisconsin can turn around a "stat" whole-genome in about 48 to 72 hours, Dimmock says, and a typical exome takes about 3 weeks. Stephen Kingsmore, president and Chief Operating Officer of the Rady Pediatric Genomic and Systems Medicine Institute in San Diego, recently unveiled an even faster wholegenome sequencing and interpretation strategy called STATseq, which can be done in just 26 hours (6).

High-speed approaches rely on sophisticated automated computational algorithms to filter variant calls for common variants and variants that have been observed in closely related species. Yet, says Dimmock, solid clinical data are key. He cites one patient who was referred to his clinic with "tufting enteropathy"-a form of severe diarrhea. Genome-wide sequencing revealed the child had a 4-bp deletion in the gene encoding EpCAM, 1 of only 2 genes associated with that particular condition.

"Having those exact words, that the child had tufting enteropathy, meant that we knew with pretty close to 
$100 \%$ certainly that we had the right mutation," explains Dimmock, who describes that finding as "one blindingly obvious flashing lightbulb [blinking] at you going, 'choose me, choose me.'” If the physician had simply input "diarrhea," diagnosis may have proved more elusive, as there are thousands of genes associated with that symptom. "Really understanding what the physician thinks is wrong with the patient makes a huge difference in the ability to make a diagnosis," Dimmock says.

The resulting diagnoses are not always actionable, of course-these are genetic defects, after all. Maybe 5\% of patients in Dimmock's clinic receive a diagnosis that is then treatable with a specific therapy, he says, though diagnoses more frequently lead to changes in management, for example, in electing for palliative care. A 2015 analysis of 35 infants subjected to STATseq by Kingsmore's team (then at Children's Mercy-Kansas City), obtained diagnoses in 20 cases (57\%), $65 \%$ of which were deemed "clinically useful" (7). In one case, a hypoglycemic child was diagnosed with ABCC8-associated familial hyperinsulinism type 1 , resulting in a targeted surgical intervention and resolution of the hyperinsulinemia.

Yet even when nothing can be done, the peace of mind that comes from parents having a definitive diagnosis cannot be overstated, says Dimmock. And about 50\% of patients could theoretically benefit from drugs that influence, say, exon-skipping or premature termination codon readthrough, provided there was time to test whether they work for the patient's condition.

But that's only true if a diagnosis can be made. Dimmock says he can make a diagnosis about one time in five-though the odds improve if the patient is not in the midst of a "diagnostic odyssey" that already has eliminated many likely candidate genes. A recent story in the Milwaukee JournalSentinel on the case of Nic Volker, one of the first children to be cured of a condition by whole-exome sequenc- ing, suggests that whole-exome sequencing yields a diagnosis in perhaps a quarter of cases (8).

Fundamentally, researchers simply have no way to predict what most variants will do. One possible approach is brute force; systematically make every possible variant in a gene and test activity - a strategy Akey's colleague Jay Shendure recently applied to BRCA1 (9). The alternative is chipping away at individual variants and conditions.

Either way, it will be a tough slog. "But," says Kingsmore optimistically, "eventually we'll get there, one SNP at a time."

\section{References}

1. 1000 Genomes Project Consortium. 2015. A global reference for human genetic variation. Nature. 526:68-74.

2. Claussnitzer, M., et al. 2015. FTOObesity variant circuitry and adipocyte browning in humans. New Engl J Med. 373:895-907.

3. Halvorsen, M., et al. 2010. Diseaseassociated mutations that alter the RNA structural ensemble. PLoS Genetics. 6:e1001074.

4. Wan, Y., et al. 2014. Landscape and variation of RNA secondary structure across the human transcriptome. Nature. 505:706709.

5. Wang, G., et al. 2014. Modeling the mitochondrial cardiomyopathy of Barth syndrome with induced pluripotent stem cell and heart-on-chip technologies. Nat Med. 20:616-623.

6. Miller, N.A., et al. 2015. A 26-hour system of highly sensitive whole genome sequencing for emergency management of genetic diseases. Genome Medicine. 7:100.

7. Willig, L K., et al. 2015. Whole-genome sequencing for identification of Mendelian disorders in critically ill infants: A retrospective analysis of diagnostic and clinical findings. Lancet Respir Med. 3:377-387.

8. Johnson, M. and K. Gallagher. 25 Oct 2015. Young patient faces new struggles years after DNA sequencing. Milwaukee Journal Sentinel. Available from: www. jsonline.com/news/health/young-patientfaces-new-struggles-years-after-dnasequencing-b99602505z1-336977681.html

9. Starita, L.M., et al. 2015. Massively parallel functional analysis of BRCA1 RING domain variants. Genetics, 200:413-422.

Written by Jeffrey Perkel, Ph.D. 四

BioTechniques 59:262-267 (November 2015) doi: 10.2144/000114350

\section{GEL-FREE OR LOW INPUT, HERD TOGETHER.}

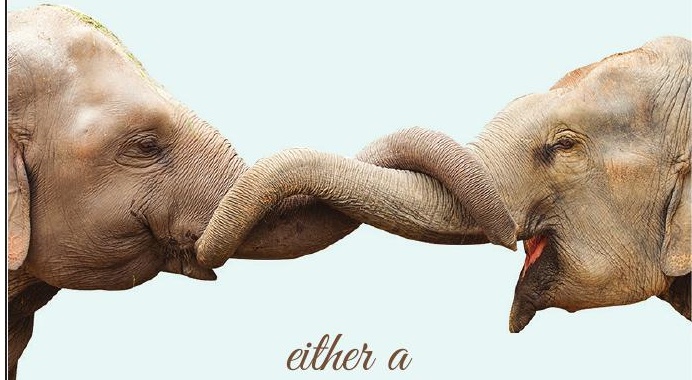

$$
\begin{gathered}
\text { Gel-Free Protocol } \\
\text { ar an } \\
\text { Input of Only } 5 \mathrm{ng} \\
\text { bath with } \\
\text { Reduced Bias }
\end{gathered}
$$

With gel purification steps, high input requirements, and inherent bias introduced during adapter ligation, small RNA library prep has always been difficult. However, with the NEXTflex ${ }^{\text {Tim }}$ Small RNA-Seq Kit v3, you can construct Illumina-compatible small RNA-Seq libraries from as little as $5 \mathrm{ng}$ of total RNA, or go completely gel-free with $100 \mathrm{ng}$. With the incorporation of randomized adapters, ligation bias is reduced, resulting in more accurate data.

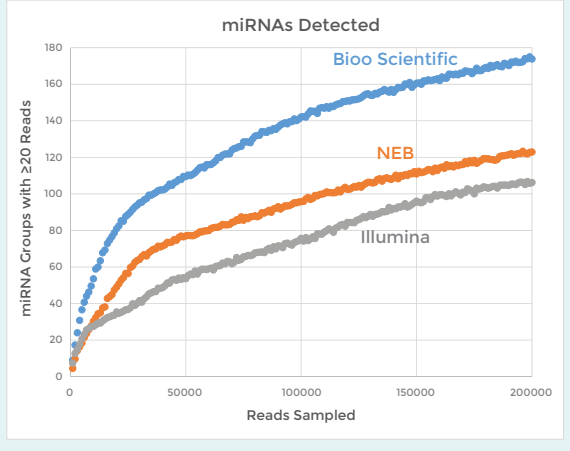

Detect more miRNAs per sequencing run than with competitor library prep kits.

Plod out your Small RNA experiment at BiooScientific.com/SmallRNA 